\title{
Structural Abnormalities are Similar in Familial and Nonfamilial Mesial Temporal Lobe Epilepsy
}

\author{
Fabio Thadeu Ferreira, Eliane Kobayashi, Iscia Lopes-Cendes, Fernando Cendes
}

\begin{abstract}
Background/Objective: Diffuse temporal lobe abnormalities can be observed on MRI of patients with mesial temporal lobe epilepsy (MTLE). Our objective was to perform qualitative and quantitative analyses of temporal lobe structures in patients with familial MTLE (FMTLE) and nonfamilial MTLE. Methods: Two groups of patients were ascertained: 67 FMTLE patients (14 with refractory seizures) and 30 patients with nonfamilial refractory MTLE. We performed qualitative analyses of MRI (with multiplanar reconstruction) and volumes of hippocampi and anterior temporal lobes in all patients, and in a normal control group of 23 individuals. We used the Chi-square test and ANOVA for statistical analyses. Results: We identified anterior temporal lobe abnormalities by visual analysis in only $4 \%$ of FMTLE patients and atrophy of the anterior temporal lobe by volumetric analysis in $19 \%$. In the group of nonfamilial MTLE patients we found anterior temporal lobe abnormalities by visual analysis in $17 \%$ of patients and anterior temporal lobe atrophy in $13 \%$. Hippocampal atrophy was present in $90 \%$ of FMTLE and in $83 \%$ of nonfamilial MTLE. No signs of cortical dysplasia were observed. Conclusion: Anterior temporal lobe atrophy and other abnormalities outside the mesial portion of temporal lobes were infrequent in both familial and nonfamilial MTLE patients. Despite the genetic basis, hippocampal atrophy in FMTLE is not associated with other abnormalities outside the mesial temporal regions.
\end{abstract}

RÉSUMÉ: Anomalies structurales similaires dans l'épilepsie du lobe temporal mésial familiale et non familiale. Introduction/Objectif: On peut observer des anomalies diffuses du lobe temporal à l'IRM de patients atteints d'épilepsie du lobe temporal mésial (ÉLTM). Le but de cette étude était d'effectuer des analyses qualitatives et quantitatives des structures du lobe temporal chez des patients atteints d'ÉLTM familiale (ÉLTMF) et non familiale (ÉLMT). Méthodes: Deux groupes de patients ont été identifiés: 67 patients atteints d'ÉLMTF, dont 14 avaient des crises réfractaires au traitement, et 30 patients atteints d'ÉLMT non familiale réfractaire. Nous avons effectué des analyses qualitatives de l'IRM (avec reconstruction multidimensionnelle) et du volume de l'hippocampe et des lobes temporaux antérieurs chez tous les patients et chez un groupe de témoins normaux comprenant 23 individus. Nous avons utilisé le test du c2 et l'ANOVA comme analyses statistiques. Résultats: Nous avons identifié des anomalies du lobe temporal antérieur par analyse visuelle chez seulement $4 \%$ des patients atteints d'ÉLTMF et une atrophie du lobe temporal antérieur par analyse volumétrique chez $19 \%$ de ces patients. Dans le groupe de patients atteints d'ÉLTM non familiale, nous avons observé des anomalies du lobe temporal antérieur par analyse visuelle chez $17 \%$ des patients et une atrophie du lobe temporal antérieur chez $13 \%$. Une atrophie de l'hippocampe était présente chez $90 \%$ des patients atteints d'ÉLTMF et chez $83 \%$ des patients atteints de la forme non familiale. Aucun signe de dysplasie corticale n'a été observé. Conclusion: Une atrophie du lobe temporal antérieur et d'autres anomalies ne touchant pas la partie mésiale des lobes temporaux étaient rares tant chez les cas familiaux que chez les cas non familiaux. Malgré son étiologie génétique, l'atrophie de l'hippocampe n'est pas associée à des anomalies de structures en dehors des régions temporales mésiales dans l’ÉLTMF.

Can. J. Neurol. Sci. 2004; 31: 368-372

Mesial temporal lobe epilepsy (MTLE) is frequently associated with mesial temporal sclerosis $^{1,2}$ which can be identified by magnetic resonance imaging (MRI) as hippocampal atrophy usually associated with abnormal T2 hyperintense signal and other abnormalities in the mesial temporal lobe structures. ${ }^{3-5}$ Pathological and MRI studies in surgical series of patients with
From the Departments of Neurology (EK, FC) and Medical Genetics (IL-C), Faculdade de Ciências Médicas, Campinas State University, Campinas, São Paulo, Brazil. FTF is a medical student.

ReCEIVED MARCh 21, 2003. ACCEPTED InfinALFORM JANUARY 15, 2004. Reprint requests to: Fernando Cendes, Departamento de Neurologia, FCM - UNICAMP, Campinas SP, Brazil, CEP13083-970 
MTLE have also described reduced volumes and abnormal structural findings outside the mesial temporal regions. ${ }^{6-12}$. One reasonable hypothesis is that more severe hippocampal atrophy is associated with more refractory seizures and more widespread abnormalities.

A positive family history of epilepsy is frequently observed among patients with MTLE. However, these families cannot be segregated to a single group. The detailed characterization of other affected family members is required for definition of a familial epilepsy syndrome. ${ }^{13-20}$

In FMTLE, the majority of affected individuals have a benign clinical presentation but some patients may have poor seizure control. ${ }^{19,20}$ Hippocampal atrophy is observed in patients with good outcome, and even in asymptomatic family members. ${ }^{21,22}$ These are strong indicators of the genetic factors in the genesis of hippocampal pathology in these families. It is not known, however, if this genetic background would be associated with more widespread temporal lobe abnormalities.

The objective of this study was to assess differences in morphology and morphometry of temporal lobe structures in patients with FMTLE and nonfamilial MTLE.

\section{Patients And methods}

We studied two groups of patients: FMTLE patients and refractory MTLE patients with no family history of epilepsy. All individuals underwent our MRI protocol after signing an informed consent approved by our Ethics Committee.

The FMTLE patients were selected from our previously published series. ${ }^{20,21}$ FMTLE was defined when at least two individuals (first-degree relatives) had a clinical-EEG diagnosis of MTLE. Exclusion criteria were: any affected family member with partial epilepsy of an extra-temporal pattern, lateral temporal lobe epilepsy and families that could not be adequately investigated (even if two individuals had diagnosis of temporal lobe epilepsy). ${ }^{20}$ For this study we included only patients with FMTLE with typical semiology of MTLE and who had clear and consistent interictal EEG spikes on mid-inferomesial temporal lobe regions, and no EEG abnormalities elsewhere.

A group of 30 randomly selected nonfamilial, clinically and electrographically well-defined refractory MTLE patients, from our epilepsy clinic, was studied for comparison. For this group of nonfamilial MTLE we included only patients we were certain that they had no family history of seizures.

A normal control group composed by 23 healthy adult volunteers from our laboratory (seven men, mean age of 28 years $(20$ - 40)) was used for determination of normal volume parameters for volumetric studies and for the morphology of temporal lobe structures by visual analysis.

Magnetic resonance images (MRI) were performed in a $2 \mathrm{~T}$ scanner (Elscint Prestige ${ }^{\circledR}$, Haifa, Israel), with $\mathrm{T} 1$ and $\mathrm{T} 2$ acquisitions in three orthogonal planes. The MRI acquisition parameters were: (1) Sagittal T1 spin echo, 6mm thick, flip angle $=180^{\circ}$; repetition time $(\mathrm{TR})=430$, echo time $(\mathrm{TE})=12$, matrix 200X350, field of view $(\mathrm{FOV})=25 \mathrm{X} 25 \mathrm{~cm}$; (2) Coronal images, perpendicular to the long axis of hippocampus, defined on the sagittal images: (a) T2-weighted and proton density "fast spin echo" (FSE), 3mm thick, flip angle $=160^{\circ}$; TR=4800, $\mathrm{TE}=18 / 108$, matrix $252 \mathrm{X} 320, \mathrm{FOV}=18 \mathrm{X} 18 \mathrm{~cm}$; (b) T1-weighted inversion recovery (IR), $3 \mathrm{~mm}$ thick, flip angle $=200^{\circ}$; $\mathrm{TR}=2800$, $\mathrm{TE}=14$, inversion time $(\mathrm{TI})=840$, matrix $130 \mathrm{X} 256$, FOV $=16 X 18 \mathrm{~cm}$; (3) Axial images parallel to the long axis of the hippocampi: (a) T1-weighted gradient echo, 3mm thick, flip angle $=70^{\circ}, \mathrm{TR}=200, \mathrm{TE}=5$, matrix $180 \mathrm{X} 232, \mathrm{FOV}=22 \mathrm{X} 22 \mathrm{~cm}$; (b) T2-weighted FSE, 4mm thick, flip angle $=120^{\circ}, \mathrm{TR}=6800$, $\mathrm{TE}=129$, matrix 252X328, FOV=21X23cm; (4) T1-weighted 3D gradient echo with voxel size of approximately $1 \mathrm{~mm}(1 \mathrm{~mm}$ thick, flip angle $=35^{\circ} ; \mathrm{TR}=22, \mathrm{TE}=9$, matrix 256X220, $\mathrm{FOV}=25 \mathrm{X} 23 \mathrm{~cm})$, acquired in the sagittal plane for multiplanar reconstruction (MPR).

Visual and volumetric analyses were performed without knowledge of clinical data. Visual analysis was performed in a workstation (OMNIPRO ${ }^{\circledR}$, Philips Medical System), using all $2 \mathrm{D}$ sequences and the $1 \mathrm{~mm}$ thick T1-gradient echo 3D sequence with MPR in the three orthogonal planes and curved reconstructions when necessary, to avoid partial volume effect. We paid special attention to the integrity of temporal lobe structures, gyral pattern, grey-white matter distinction and signal intensity. We analyzed separately the anatomy and signal intensity in the mesial portion of temporal lobe (defined here as all structures medial to the fusiform gyrus) and basolateral temporal areas.

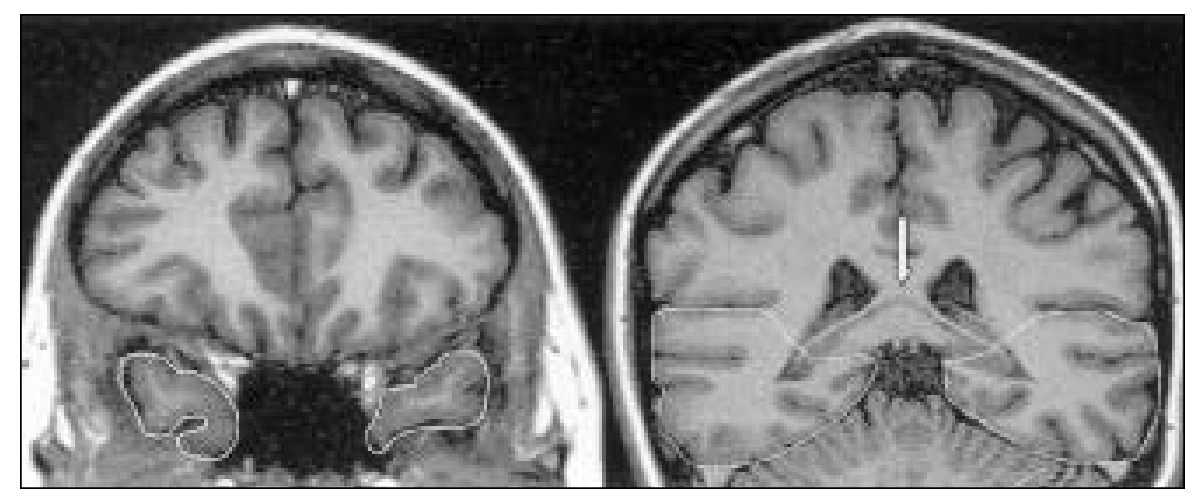

Figure 1: Boundaries for determination of temporal lobe volumes, from the first slice (at left) showing the temporal poles throughout the temporal lobes until the identification of the crura fornix (arrow). Volumes were corrected by the variation in total brain volume. 


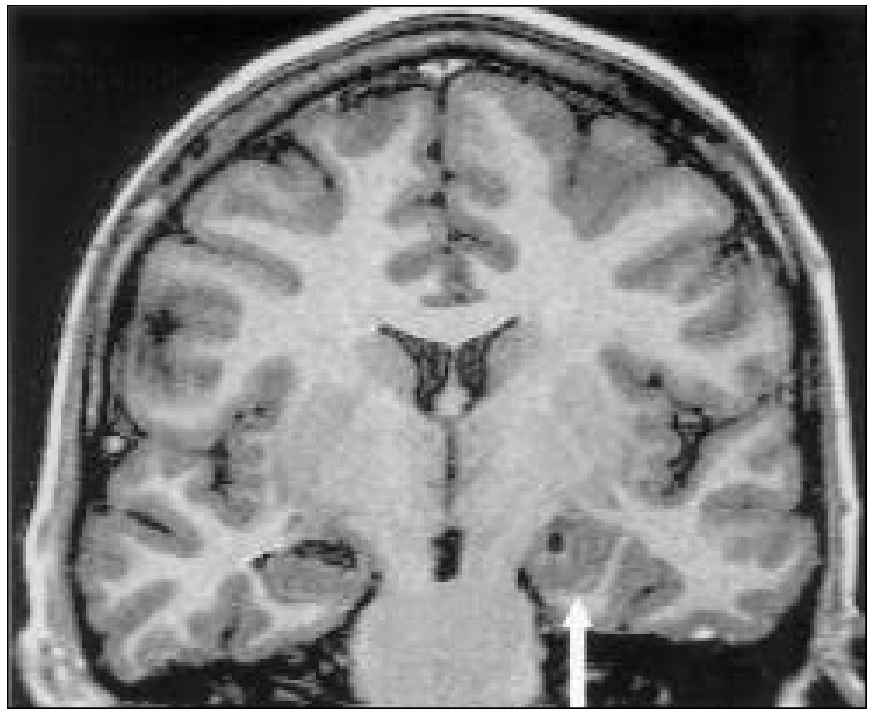

Figure 2: Left hippocampal malformation, characterized by abnormal shape and orientation (vertical orientation of the left hippocampal axis) associated with enlarged left fusiform gyrus and deep collateral sulcus with thickened cortex (arrow).

Volumetric measurements were performed by manual delineation of temporal lobes and hippocampi, using NIH Image Program $^{\circledR}$ (http://rsb.info.nih.gov/nih-image) in T1-IR coronal slices. For temporal lobe volumes (TLV), we included the boundaries of cortical surface from the temporal lobes, from the first slice presenting the temporal poles until the slice where we could identify the crura fornix (Figure 1). Hippocampal volumes were determined according to anatomic guidelines from a standard protocol. ${ }^{23}$ Values were corrected by the variation in intracranial volume, which was also manually delineated from the temporal poles to the last segmented slice, excluding the brain stem.

We determined the TLV and hippocampal volumes, as well as the asymmetry index (AI), for each subject. The AI was defined as the ratio of the smaller by the larger structure for each individual (AI for TLV and AI for hippocampal volumes). Atrophy was defined as values below two standard deviations from the mean of the control group.

For statistical analyses we used SYSTAT ${ }^{\circledR}$. We used analysis of variance (ANOVA) to assess differences in mean volumes between groups. The frequency of anterior temporal lobe atrophy and of hippocampal atrophy among groups was evaluated by the Fisher's and Chi-square tests. For correlation of TLV and hippocampal volumes we used Pearson's simple correlation or regression analysis.

\section{RESUltS}

A total of 97 patients were studied. There were 67 FMTLE patients: 14 with refractory seizures and 53 with well-controlled seizures or remission of epilepsy; from 30 unrelated families (21 men and 46 women) with mean age of 35 years ( 8 to 76 years). Most of these patients have been included in previous publications. $^{20,21}$ In addition, there were 30 nonfamilial refractory MTLE patients: 13 men and 17 women, mean age of 34 years (15 to 55 years).

There was no difference in age at evaluation between the three groups of patients: benign FMTLE, refractory FMTLE and nonfamilial refractory MTLE (ANOVA, $\mathrm{p}=0.79$ ). Seizure semiology of patients with FMTLE was similar as compared with patients with nonfamilial MTLE. ${ }^{20,21}$ Febrile seizures in childhood were reported in five (7\%) patients with FMTLE and four (13\%) with nonfamilial MTLE (Chi-square, $\mathrm{p}=0.6$ ).

Five patients with FMTLE underwent anterior temporal lobe resection including amygdala and hippocampus. Qualitative pathological studies showed classical mesial temporal sclerosis pattern in three patients. No significant abnormalities were observed due to insufficient hippocampal specimens referred to pathology in the other two patients. No abnormalities in the temporal poles were observed in routine histopathology. Twentyseven of the 30 patients with nonfamilial refractory MTLE underwent surgery. Postoperative histopathology showed various degrees of hippocampal neuronal loss and gliosis with typical pattern of hippocampal sclerosis in all of them. None of them had signs of heterotopic neurons or cortical dysgenesis in the

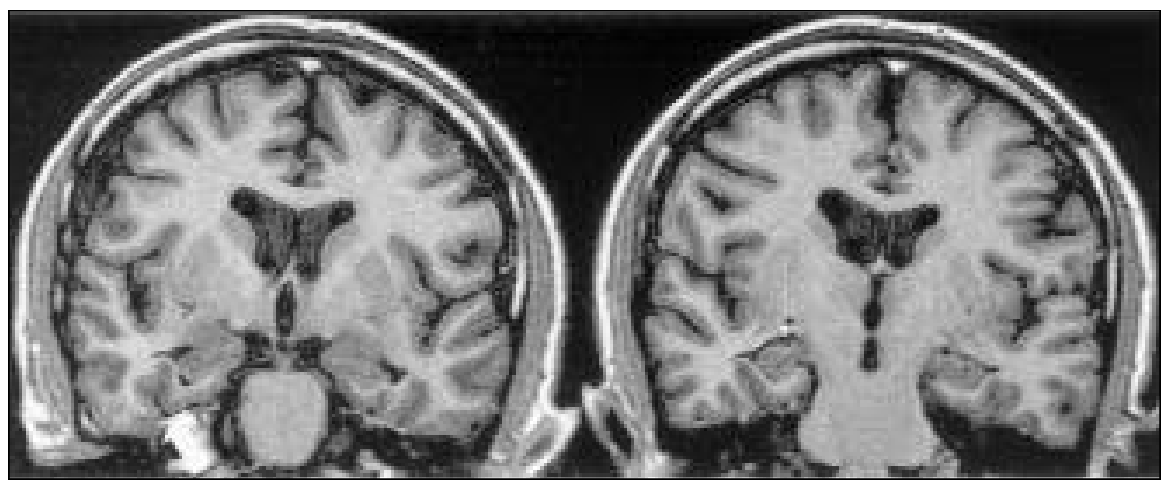

Figure 3: Abnormalities in the right medial temporal region in a patient with benign FMTLE, including abnormal shape and width of parahippocampal and fusiform gyri and collateral sulcus (large arrow) associated with an ipsilateral rounded hippocampus, which was atrophic by volumetric measurements (small arrow). 
surgical specimens. In two patients there was additional nonspecific gliosis in the white matter on the fragments of anterior temporal lobe.

Magnetic resonance image abnormalities in the anterior and lateral temporal lobe regions were found in only 3/67 (4\%) FMTLE patients. One had left temporal lobe hypogenesis (reduced size with no abnormal signal intensity) ${ }^{24}$ associated with an arachnoid cyst; one had a small left temporal lobe encephalocele; and one had a non-specific gliotic lesion in the pole of the right temporal lobe. In the group of nonfamilial MTLE patients, visually determined abnormalities were observed in 5/30 (17\%) patients and consisted of small hyperintense white matter lesions in the anterior temporal lobes. There was no relationship with the presence of hyperintense T2 lesions and age or severity of epilepsy (ANOVA, $\mathrm{p}>0.5$ ).

Mesial temporal lobe structures abnormalities on MRI were observed in both groups of patients. We found abnormal shape/orientation of parahippocampal gyrus, fusiform gyrus and collateral sulcus (in association with hippocampal atrophy or abnormally shaped and oriented hippocampus) in $12(18 \%)$ FMTLE patients (Figures 2 and 3). In nonfamilial MTLE patients, such abnormalities were found in 3/30 (10\%) patients, with altered shape of collateral sulcus associated with an enlarged and distorted fusiform gyrus (two bilateral, one left).

Anterior temporal lobe atrophy was found in 13 of $67(19 \%)$ patients with FMTLE and in 4 of $30(13 \%)$ patients with nonfamilial MTLE $\left(\chi^{2}, \mathrm{p}=0.5\right)$. Hippocampal atrophy was observed in 60 of $67(90 \%)$ patients with FMTLE (87\% patients with benign FMTLE, and in all 14 patients with refractory FMTLE); whereas 25 of $30(83 \%)$ patients with nonfamilial MTLE had hippocampal atrophy $\left(\chi^{2}, \mathrm{p}=0.28\right)$.

Temporal lobe volumes and hippocampal volumes were significantly different between controls and both groups of patients (familial and nonfamilial) (ANOVA, and Tukey posthoc comparisons; $\mathrm{p}<0.05)$; however there was no significant difference in the pair-wise comparisons between familial and nonfamilial MTLE patients ( $\mathrm{p}>0.1$ ).

Correlation analyses showed that TLVs were associated with ipsilateral hippocampal volumes $\left(r^{2}=0.073, p<0.001\right)$ but not with contralateral hippocampus $\left(\mathrm{r}^{2}=0.001, \mathrm{p}=0.7\right)$, although less than $10 \%$ of the variance in TLVs could be related to the hippocampal volumes, as indicated by the $\mathrm{r}^{2}$ value. Hippocampal volumes did not differ between patients with anterior temporal lobe atrophy and with normal TLVs (ANOVA, $\mathrm{p}=0.08$ ).

\section{DISCUSSION}

In FMTLE, the presence of hippocampal atrophy in patients with well-controlled epilepsy and also in asymptomatic individuals $^{20-22}$ is indicative of a pre-existing structural abnormality, probably determined by genetic factors.

Visual inspection of the temporal lobes using MPR identified a few patients with minor developmental abnormalities restricted to the mesial portion, in both FMTLE and sporadic MTLE patients. The MRI signs of cortical dysplasia (thick, blurred and hyperintense cortex or white-gray matter transition) or temporal lobe developmental malformation associated with abnormal MRI signal in the white matter of temporal pole as described by Kuzniecky and colleagues, ${ }^{25,26}$ were not observed in our series.
Hyperintense white matter lesions in the anterior temporal lobes were found in only one of 67 FMTLE patients and in $17 \%$ of patients with nonfamilial MTLE. This is a difference that is difficult to explain, due to the "nonspecific" nature of these lesions, and also because its presence was not related to age or severity of epilepsy. However, one possible explanation for these white matter signal changes is reactive astrogliosis, as demonstrated in postoperative histopathology of two patients with nonfamilial MTLE. Therefore, this difference may indicate that environmental factors, such as trauma or infections, are more important in determining epilepsy in non-familial MTLE than in FMTLE patients.

In the present series, five patients with FMTLE who underwent surgery and histopathology did not show white matter abnormalities in the anterior temporal lobe or evidence of cortical dysgenesis. Conversely, three of these patients had neuronal loss and gliosis with the classical pattern of mesial temporal sclerosis (in two patients the hippocampal specimen was insufficient for analyses), which did not differ from postoperative histopathology of patients with nonfamilial MTLE. These findings are confirmed in recent publication that includes a series of 20 patients with FMTLE who underwent surgery. ${ }^{27}$

Interestingly, antecedent febrile seizures were less frequent among patients with FMTLE (7\%) than nonfamilial MTLE (13\%), although not statistically significant. This lower frequency of febrile seizures in childhood in FMTLE was also found in a multicenter study. ${ }^{21}$ Conversely, patients with FMTLE had more frequent abnormalities in the shape and volume of hippocampi than nonfamilial MTLE patients, but this difference was not statistically significant.

The genetic factor associated with the development of hippocampal pathology in FMTLE does not imply in a more widespread abnormality in the temporal regions. Abnormalities were observed in the mesial temporal structures and were associated with MRI signs of mesial temporal sclerosis. The presence of diffuse functional abnormalities or other pathological abnormalities undetected by conventional postoperative histopathology, however, cannot be ruled out in these families.

\section{ACKNOWLEDGEMENTS}

FTF and EK received scholarship grants from Fundação de Amparo à Pesquisa do Estado de São Paulo (FAPESP), São Paulo, Brazil.

We thank all the patients and families for their cooperation in this study.

\section{REFERENCES}

1. Hauser WA. The natural history of temporal lobe epilepsy. In: Lüders HO (Ed). Epilepsy Surgery. New York: Raven Press, 1992:133-141.

2. Gloor P. Mesial temporal sclerosis: historical background and an overview from a modern perspective. In: Lüders $\mathrm{H}$, (Ed). Epilepsy surgery. New York: Raven Press, 1991: 689-703.

3. Jackson GD, Berkovic SF, Tress BM, et al. Hippocampal sclerosis can be reliably detected by magnetic resonance imaging. Neurology 1990;40:1869-1875.

4. Berkovic SF, Andermann F, Olivier A, et al. Hippocampal sclerosis in temporal lobe epilepsy demonstrated by magnetic resonance imaging. Ann Neurol 1991;29:175-182. 
5. Watson C, Jack CR, Cendes F. Volumetric magnetic resonance imaging: clinical applications and contributions to the understanding of temporal lobe epilepsy. Arch Neurology 1997; 54: 1521-1531.

6. Cendes F, Andermann F, Gloor P, et al. MRI volumetric measurement of amygdala and hippocampus in temporal lobe epilepsy. Neurology 1993;43:719-725.

7. Jack CR, Sharbrough FW, Twomey CK, et al. Temporal lobe seizures: lateralization with magnetic resonance volume measurements of the hippocampal formation. Radiology 1990; 175:423-429.

8. Lencz T, McCarthy G, Bronen RA, et al. Quantitative magnetic resonance imaging in temporal lobe epilepsy: relationship to neuropathology and neuropsychological function. Ann Neurol 1992;31:627-629.

9. Lee JW, Reutens DC, Dubeau F, et al. Morphometry in temporal lobe epilepsy. Magn Reson Imaging 1995;13:1073-1080.

10. Marsh L, Morrel ML, Shear PK, et al. Cortical and hippocampal deficits in temporal lobe epilepsy. Epilepsia 1997; 38:576-587.

11. Briellmann RS, Jackson GD, Kalnins R, et al. Hemicranial volume deficits in patients with temporal lobe epilepsy with and without hippocampal sclerosis. Epilepsia 1998;39(11):1174-1181.

12. Breier JI, Leonard CM, Bauer RM, et al. Quantified volumes of temporal lobe structures in patients with epilepsy. J Neuroimaging 1996;6:108-114.

13. Ottman R, Risch N, Hauser WA, et al. Localization of a gene for partial epilepsy to chromosome 10q. Nat Genet 1995;10(1):5660

14. Poza JJ, Sáenz A, Martinez-Gil A, et al. Autosomal dominant lateral temporal epilepsy: clinical and genetic study of a large Basque pedigree linked to chromosome 10q. Ann Neurol 1999;45:182188.

15. Winawer MR, Boneschi FM, Barker-Cummings C, et al. Four new families with autosomal dominant partial epilepsy with auditory features: clinical description and linkage to chromosome 10q24. Epilepsia 2002;43(1):60-67.

16. Singh R, Scheffer IE, Crossland K, Berkovic SF. Generalized epilepsy with febrile seizures plus: a common childhood-onset genetic epilepsy syndrome. Ann Neurol 1999;45(1):75-81.

17. Scheffer IE, Phillips HA, O'Brien CE, et al. Familial partial epilepsy with variable foci: a new partial epilepsy syndrome with suggestion of linkage to chromosome 2. Ann Neurol 1998;44(6):890-899.

18. Berkovic SF, McIntosh A, Howell RA, et al. Familial temporal lobe epilepsy - a common disorder identified in twins. Ann Neurol 1996;40:227-235.

19. Cendes F, Lopes-Cendes I, Andermann E, Andermann F. Familial temporal lobe epilepsy: a clinically heterogeneous syndrome. Neurology 1998;50(2): 554-557.

20. Kobayashi E, Sousa SC, Lopes-Cendes I, et al. Seizure outcome and hippocampal atrophy in familial mesial temporal lobe epilepsy. Neurology 2001;56(2):166-172.

21. Kobayashi E, D'Agostino MD, Lopes-Cendes I, et al. Hippocampal atrophy and T2 weighted signal changes in familial mesial temporal lobe epilepsy. Neurology 2003; 60(3):405-409.

22. Kobayashi E, Li LM, Lopes-Cendes I, Cendes F. Magnetic resonance imaging evidence of hippocampal sclerosis in asymptomatic, first-degree relatives of patients with familial mesial temporal lobe epilepsy. Arch Neurol 2002; 59:1891-1894.

23. Watson C, Andermann F, Gloor P, et al. Anatomic basis of amygdaloid and hippocampal volume measurement by magnetic resonance imaging. Neurology 1992;42:1743-1750.

24. Robertson SJ, Wolpert SM, Runge VM. MR imaging of middle cranial fossa arachnoid cysts: temporal lobe agenesis syndrome revisited. AJNR Am J Neuroradiol 1989;10(5):1007-1010.

25. Kuzniecky R, Garcia JH, Faught E, Morawetz RB. Cortical dysplasia in temporal lobe epilepsy: magnetic resonance imaging correlations. Ann Neurol 1991;29:293-298.

26. Ho SS, Kuzniecky R, Gilliam F, et al. Temporal lobe developmental malformations and epilepsy: dual pathology and bilateral hippocampal abnormalities. Neurology 1998; 50(3): 748-754.

27. Kobayashi E, D’Agostino MD, Lopes-Cendes I, et al. Outcome of surgical treatment in familial mesial temporal lobe epilepsy. Epilepsia 2003; 44(8): 1080-1084. 